\title{
Anti-thyroid peroxidase antibody positivity and other obstetric complications in women with recurrent miscarriage
}

\author{
Sneha Jaiswal*, Tarasankar Bag
}

Department of Obstetrics and Gynecology, Medical College Kolkata, Kolkata, Bengal, India

Received: 24 October 2016

Revised: 31 October 2016

Accepted: 23 November 2016

\section{*Correspondence:}

Dr. Sneha Jaiswal,

E-mail: sneha.jais22@gmail.com

Copyright: (c) the author(s), publisher and licensee Medip Academy. This is an open-access article distributed under the terms of the Creative Commons Attribution Non-Commercial License, which permits unrestricted non-commercial use, distribution, and reproduction in any medium, provided the original work is properly cited.

\begin{abstract}
Background: Thyroid dysfunction and autoimmunity are relatively common in women of reproductive age group and has been associated with various adverse pregnancy outcomes such as recurrent miscarriage, preeclampsia and preterm labour. The aim of this study was to find out association between anti-tpo antibody and recurrent miscarriage and to evaluate obstetric complications such as preeclampsia and preterm labour in them.

Methods: This case control observational study was carried out from July 2014 to June2015 on 110 pregnant mothers out which 55 with history of recurrent miscarriage were cases and 55 without such history were controls.

Results: The mean age of the cases were 24.18 yrs. Majority of pregnancy losses occurred between 9-14wks. The prevalence of anti-tpo antibody positivity in the study population was $15.45 \%$.out of 55 cases 12 were positive for anti-tpo antibody(21.8\%).Positive anti-tpo antibody was associated with high TSH level than the negative anti tpo antibody $(\mathrm{p}<.000)$. The prevalence of preeclampsia and preterm labour in recurrent miscarriage patients were $9.09 \%$ and $5.45 \%$ respectively which was not significantly higher than background risk.1 out of 5 patients with preeclampsia had anti-tpo positive and none with preterm labour had anti-tpo antibody positivity.

Conclusions: The prevalence of thyroid autoimmunity was higher in patients with history recurrent miscarriage history than the control population. Even in women with biochemically normal thyroid function there was relationship between recurrent miscarriage and thyroid autoimmunity. Thus anti-tpo antibody could be risk marker for RSM. Screening for subclinical hypothyroidism $(\mathrm{TSH}>2.5$ but $<10)$ and anti-tpo antibody can be suggested as these identify women with increased risk of miscarriage.
\end{abstract}

Keywords: Anti-tpo antibody, Hypothyroidism, Pregnancy, Preeclampsia, Preterm Labour, Recurrent Miscarriage, TSH

\section{INTRODUCTION}

Miscarriage is defined as any pregnancy ending spontaneously prior the fetus can survive. Recurrent miscarriage, defined as loss of 3 or more consecutive pregnancies affects $1 \%$ of couples trying to conceive. The etiology of recurrent spontaneous miscarriage includes epidemiological factors such as maternal age and number of previous miscarriages, anti-phospholipids syndrome, genetic factors, anatomical factors, endocrine factors, immune factors; inherited thrombophillic defects. ${ }^{1}$ Women with RPL can be classified into three separate groups, as primary, secondary and tertiary aborters. Primary aborters are women with no previous live birth, secondary aborters if there was a live birth followed by pregnancy losses, and tertiary aborters are women who had pregnancy losses followed by a live birth. ${ }^{2}$

Autoimmune thyroid disease (AITD) is by far the most frequent cause of Hypothyroidism in women of reproductive age. Thyroid disorders have been long suspected to cause early pregnancy loss and other adverse pregnancy outcomes. Although the worst overt 
hypothyroidism is infrequent in pregnancy, subclinical hypothyroidism has an incidence of $2-3 \% .^{3}$

Autoimmune thyroid diseases are characterized by the presence of antithyroid antibodies, specifically anti thyroid peroxidise (TPO-abs) and anti-thyroglobulin (TG-abs).

Women in euthyroid state but with thyroid autoimmunity are twice likely to experience spontaneous miscarriages ${ }^{4}$ as it probably represents a generalized activation of immune system, or there is an increased risk of progression to subclinical hypothyroidism or probably due to transplacental transfer of Thyroid receptor blocking antibodies. Hence screening for subclinical hypothyroidism and thereby anti-tpo antibody might be required for patients with history of recurrent miscarriage. The present study was carried out to find out relation between anti-tpo antibody and in RSM mothers without overt hypothyroidism and to evaluate other obstetric complications in them.

\section{METHODS}

This case control observational study was carried out in the Department of Obstetrics and Gynaecology, Eden Hospital, Medical College; Kolkata between $1^{\text {st }}$ July 2014 - 30 ${ }^{\text {th }}$ June 2015. The study was approved by ethical committee. All patients provided written informed consent.

55 women who presented with history of recurrent miscarriage were taken as cases \&55 mothers without such history were taken as controls. Those with anatomic uterine defects, overt hypothyroidism, antiphospholipids syndrome and other autoimmune diseases were excluded from cases.

Under quality control and safety procedures for sample collection $10 \mathrm{ml}$ venous blood sample was collected in vaccutainer tubes. Serum samples were sent for anti-TPO and TSH assay. Serum TSH levels were determined using Micro particle Enzyme Immunoassay (MEIA) kits.

Normal reference range in pregnancy for TSH: 0.35 $2.5 \mu \mathrm{IU} / \mathrm{ml}$ (as per our hospital reference value), values $>2.5 \mu \mathrm{IU} / \mathrm{ml}$ were considered high for pregnancy. AntiTPO antibodies quantitative determination was done using CLIA (Chemiluminescemt Immunoassay) kits.

TPO levels >34 iu/ml were considered abnormal and these women were considered TPO+VE. Cases $\backslash$ were evaluated for obstetrics complications such as preeclampsia and preterm labour.

\section{RESULTS}

Analysis of statistical data was done by standard statistical tools used for epidemiologic statistics. In this study, a chi-square test was used to test the association between different diagnostic groups and various categorical variables. In all cases, two-tailed tests were used, and $\mathrm{P}<0.05$ was considered statistically significant. Exhaustive chi-square automatic interaction detection (CHAID) was utilized to identify significant difference, discriminating the two study groups. Statistical analysis was done using SPSS software (version 20.0) for Windows.

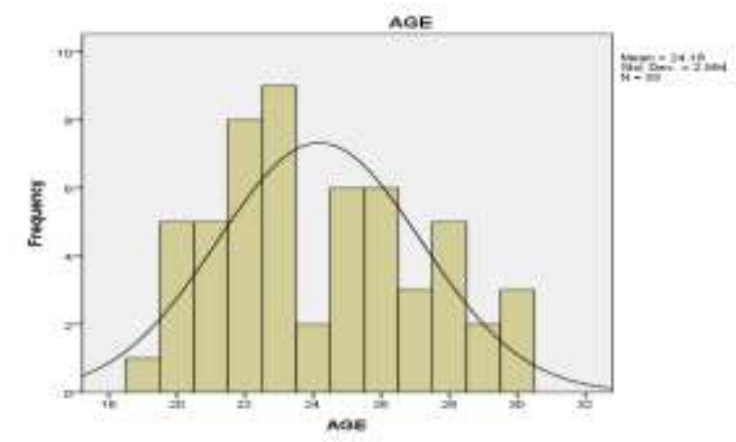

Figure 1: Histogram showing the distribution of age among cases $(n=55)$.

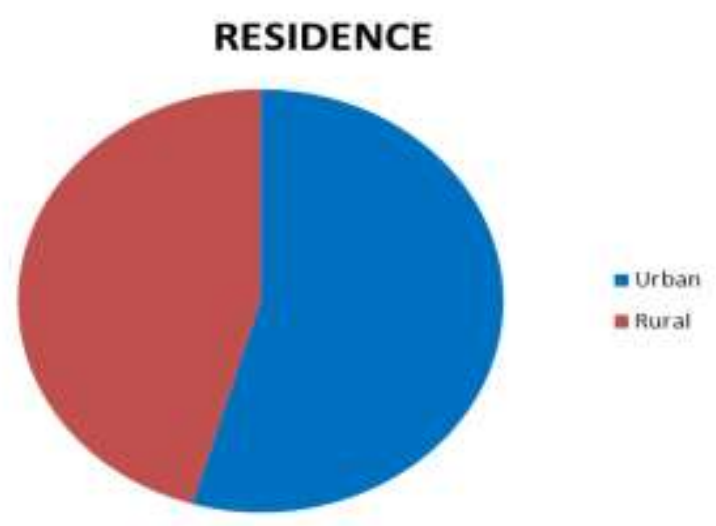

Figure 2: Pie diagram showing distribution of cases according to residence $(n=55)$.

\section{Socio Economic Status}

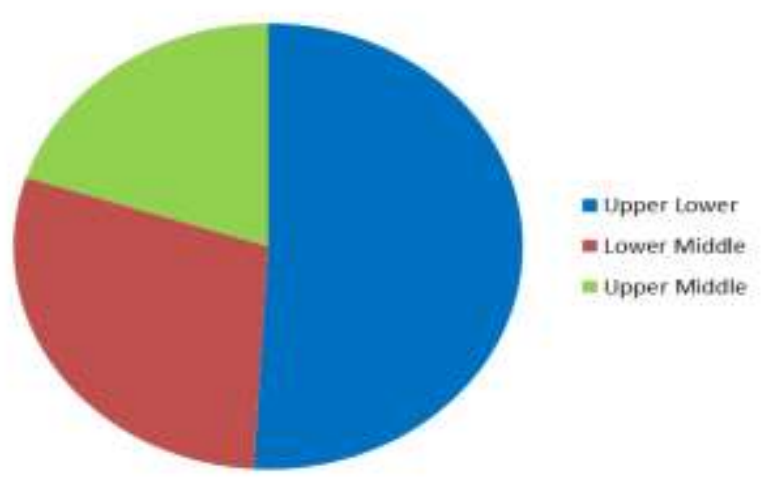

Figure 3: Pie diagram showing distribution of cases according to their Socio Economic Status (SES) as per modified Kuppuswamy scale $(\mathbf{n}=55)$. 


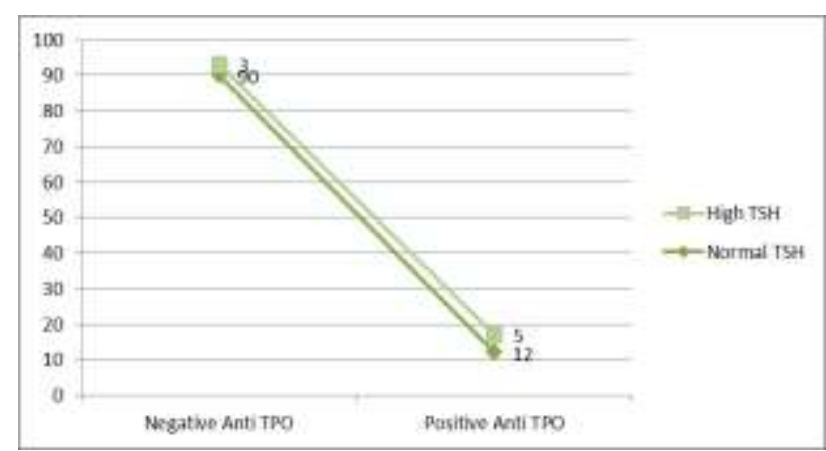

Figure 4: Correlation between Anti TPO status and TSH level.

The basic demographic variables showed mean age of cases was 24.18 years (Figure 1), $54.5 \%$ were from urban areas (Figure 2) and 50.9\% were from upper lower class (Figure 3). Out of 55 cases 12 were anti tpoAb +ve
(21.81\%) and $7.3 \%$ had subclinical hypothyroidism. The prevalence of tpo positivity in the control group was $9.09 \%$. The odd ratio of having anti-tpoAb times higher in RSM groups than controls but this association is not statistically significant $(\mathrm{p}=0.065)$ as Chi square is 3.409 (Table 1). Correlation analysis was done between TSH and Anti TPO status, and the correlation coefficient found $0.364, \mathrm{p}$ value $<0.000$ which is significant at 0.01 level. It means if Anti TPO become positive the status of TSH level also become higher or vice versa (Figure 4). The prevalence of preeclampsia and preterm labour in recurrent miscarriage patients were $9.09 \%$ (Table 2) and $5.45 \%$ (Table 3 ) respectively. 1 out of 5 patients with preeclampsia had anti-tpo positive and none with preterm labour had anti-tpo antibody positivity.

The odds of having anti tpoAb +ve in RSM was 2.791 times higher than in control group but this association is not statistically significant as $(\mathrm{p}=0.065) \quad$ (Table 1).

Table 1: Association between anti-TPO antibody status with RSM.

\begin{tabular}{|c|c|c|c|c|c|}
\hline \multicolumn{6}{|c|}{ Anti-TPO *recurrent miscarriage cross tabulation } \\
\hline & & & \multicolumn{2}{|l|}{ Anti-TPO } & \multirow{2}{*}{ Total } \\
\hline & & & Negative & Positive & \\
\hline \multirow{4}{*}{ RSM } & \multirow{2}{*}{ Absent } & Count & 50 & 5 & 55 \\
\hline & & $\%$ within RSM & $90.9 \%$ & $9.09 \%$ & $100.0 \%$ \\
\hline & \multirow{2}{*}{ Present } & Count & 43 & 12 & 55 \\
\hline & & \% within RSM & $78.18 \%$ & $21.82 \%$ & $100.0 \%$ \\
\hline \multirow{2}{*}{ Total } & & Count & 93 & 17 & 110 \\
\hline & & \% within RSM & $84.54 \%$ & $15.45 \%$ & $100.0 \%$ \\
\hline
\end{tabular}

Odds ratio $2.791, \mathrm{p}=0.065$

In our study the mean age of cases 24.18 years, SD 2.994, range 19-30 years (Figure 1).

Table 2: Distribution of preeclampsia in the case population $(\mathbf{n}=55)$.

\begin{tabular}{|ll|l|}
\hline Preeclampsia & Frequency & Percent \\
\hline Present & 5 & $9.09 \%$ \\
\hline Absent & 50 & $90.91 \%$ \\
\hline
\end{tabular}

$54.5 \%$ of the cases were from urban population and $45.5 \%$ were from rural population (Figure 2 ).

$50.9 \%$ cases were from upper lower, $29.1 \%$ were from lower middle and $20.0 \%$ were from upper middle class (Figure 3).

Correlation analysis has been done among TSH and Anti TPO status, and the correlation coefficient found $0.364, p$ value $<.000$ which is significant at 0.01 level. It means if Anti TPO become positive the status of TSH level also become higher or vice versa (Figure 4).
Table 3: Distribution of preterm labour in the case population $(n=55)$.

\begin{tabular}{|lll|}
\hline Preterm labour & Frequency & Percent \\
\hline Present & 3 & $5.45 \%$ \\
\hline Absent & 52 & $94.54 \%$ \\
\hline
\end{tabular}

\section{DISCUSSION}

In our study, $15.45 \%$ pregnant mothers $(21.81 \%$ cases and $9.09 \%$ controls) were anti-TPO positive, finding similar with the literature which stated thyroid antibody positivity in $5-15 \%$ of the women during reproductive age group. ${ }^{5,6}$

The odd ratio of having anti-tpo $\mathrm{Ab}+\mathrm{ve}$ was 2.71 times higher in RSM groups than controls. Our results were in agreement with other researchers who found relationship between anti-TPO positivity and RSM. Stagnaro -Green and colleagues reported a doubling of the miscarriage rate in euthyroid pregnant women with positive thyroid antibodies. $^{7}$ 
Similar results were found in a case control study in Greek population. ${ }^{8}$

The prevalence of subclinical hypothyroidism was higher in tpo $\mathrm{Ab}+\mathrm{ve}$ group than tpoAb-ve group (62.5\% vs $37.5 \%$ ). Bagis et al., found a significantly higher TSH among anti-thyroid antibodies positive group compared to those who were negative. ${ }^{9}$

In our study 5 RSM cases had preeclampsia out of which only 1 pt had tpo $\mathrm{Ab}+\mathrm{ve}$ and 3 had preterm labour but none had anti tpo Ab+ve. Our findings were similar to that of Abbassi-Ghanavati and associates who did not find any increased risk for preterm birth in TPO positive women compared with the risk in euthyroid women with TPOAb -ve. ${ }^{10}$

Our results of preeclampsia and preterm labour may be due to very less numbers. It could have been overcome if sample size becomes larger.

Few investigators have also shown benefit of levothyroxine treatment in women with RSM with TpoAb +ve. ${ }^{11}$

In our study the prevalence of thyroid autoimmunity was higher in women with history of RSM than those with uneventful pregnancy history again those with tpo $\mathrm{Ab}+\mathrm{ve}$ have higher prevalence of subclinical hypothyroidism (2.5mIU/L $>\mathrm{TSH}<10 \mathrm{mIU} / \mathrm{L})$.In conclusion, thyroid autoimmunity and subclinical hypothyroidism can be considered as risk marker for RSM, treatment with levothyroxine which needs more need further studies might help to reduce the risk. Euthyroid women in early stages of pregnancy with tpo $A b+v e$ are still at risk of developing hypothyroidism later; therefore their serum TSH levels should be monitored.

Funding: No funding sources Conflict of interest: None declared

Ethical approval: The study was approved by the Institutional Ethics Committee

\section{REFERENCES}

1. Green-top Guidelines no.17, April 2011. Available from: https://www.rcog.org.uk/globalassets/ documents/guidelines/gtg_17.pdf.

2. Mowbray JF, Underwood JL. Abstracts of Contributors' Individual Data Submitted to the Worldwide Prospective Observation Study on Immunotherapy for Treatment of Recurrent Spontaneous Abortion. AJRI. 1994;32:261-74.

3. Casey BM, Dash JS, Wells CE. Subclinical hypothyroidism and pregnancy outcomes. Obstet Gynecol. 2005;105(2):239.

4. Faussett MB, Branch DW. Autoimmunity and pregnancy loss. Semin Reprod Med. 2000;18:37992.

5. Feki M, Omar S. Thyroid disorders in pregnancy: frequency and association with selected diseases and obstetrical complications in Tunisian women. Clinical Biochemistry. 2008;41(12):927-31.

6. Allan WC, Haddow JE, Palomaki GE. Maternal thyroid deficiency and pregnancy complications: implications for population screening. Journal of Medical Screening. 2000;7(3):127-30.

7. Stagnaro-Green A, Roman SH, Cobin RH. Detection of at risk pregnancy by means of highly sensitive assays for thyroid antibodies. J Am Med Assoc. 1990;264:1422-5.

8. Ticconi C, Giuliani E, Veglia M, Pietropolli A, Piccione E, Di Simone N. Thyroid autoimmunity and recurrent miscarriage. Am J Reprod Immunol. 2011;66(6):452-9.

9. Bagis T, Gokcel A, Saygili ES. Autoimmune thyroid disease in pregnancy and the postpartum period: relationship to spontaneous abortion. Thyroid. 2010;11:1049-53.

10. Abbassi-Ghanavati M, Casey B, Spong C. Pregnancy outcomes in women with thyroid peroxidase antibodies. Obstetrics Gynaecology. 2010;116(2 Pt 1):381.

11. Negro R, Mangieri T, Coppola L, Presicce G. Levothyroxine treatment in thyroid peroxidase antibody-positive women undergoing assisted reproduction technologies: a prospective study. Hum Reprod. 2005;20:1529-33.

Cite this article as: Jaiswal S, Bag T. Anti-thyroid peroxidase antibody positivity and other obstetric complications in women with recurrent miscarriage. Int J Reprod Contracept Obstet Gynecol 2017;6:1903. 